\title{
Tumeur à cellules géantes : à propos d'un cas récidivant et agressif à localisation mandibulaire
}

\author{
L. de Cidrac (Paris), L. Radoï (Montrouge), R. Pecorari (Montrouge), T. Nguyen \\ (Montrouge)
}

Introduction : La tumeur à cellules géantes (TCG) est une lésion osseuse qui se développe préférentiellement au niveau de l'épiphyse des os longs chez des sujets de 20 à 40 ans, mais exceptionnellement au niveau des maxillaires. D'étiologie inconnue, elle fait partie du groupe des tumeurs osseuses bénignes. Ce groupe nosologique comprend le granulome central à cellules géantes (GCCG), le chérubisme, le kyste anévrismal ainsi que les TCG et les tumeurs brunes liées à l'hyperthyroïdie. L'histologie ne permet pas de poser un diagnostic de certitude entre la TCG et le GCCG. Cependant, les TCG présentent un tableau clinique plus agressif et récidivant. Il existe un risque de transformation maligne en sarcome dans 10 à 20\% des cas (Barthélemy 2009) et un fort potentiel métastatique (Martin-Duverneuil 2004). Observation : Le cas rapporté est celui d'une patiente de 28 ans qui présentait une tuméfaction intrabuccale douloureuse de $35 \mathrm{~mm}$ de grand axe, en distal de 47. Le Cone Beam (CBCT) montrait une lésion osseuse radioclaire sous-jacente de $22 \mathrm{~mm}$ de grand axe, à proximité d'un apex résiduel de 48 . Le diagnostic initial était celui d'un kyste résiduel compliqué d'une cellulite. Le traitement a consisté en une énucléation simple. L'examen anatomopathologique suspectait un granulome périphérique à cellules géantes (GPCG) avec atteinte osseuse. La patiente a été perdue de vue 4 mois jusqu'à la récidive de la lésion. Le nouveau CBCT montrait une lésion ostéolytique de $40 \mathrm{~mm}$ de grand axe, au niveau de l'angle mandibulaire, envahissant la branche montante avec perforation des corticales et atteinte des tissus mous. Une chirurgie interruptrice mandibulaire en marges saines avec reconstruction par attelle en titane préformée a été réalisée. L'examen anatomopathologique de la pièce d'exérèse n'a pas pu conclure entre GCCG et TCG. La patiente a été suivie 2 ans sans récidive. Discussion : Contrairement au GPCG, le GCCG et la TCG se développent d'abord dans l'os spongieux puis de manière excentrique jusqu'aux corticales osseuses qui peuvent être détruites et aux tissus mous qui sont refoulés ou envahis. Le contenu est mou, de couleur brun-rouge, parfois vacuolaire ou hémorragique. L'examen histologique montre un stroma assez homogène, très vasculaire, contenant, à côté de cellules mononuclées, de grandes cellules multinucléées : les cellules géantes. Le nombre de noyaux serait corrélé avec l'agressivité de la tumeur (Ficarra 1987). Moins fréquente que le GCCG, la TCG est plus agressive. Elle récidive dans environ $50 \%$ des cas. La recommandation actuelle est de la traiter par exérèse chirurgicale réglée avec des limites histologiques saines. Le curetage est insuffisant pour prévenir le risque de récidive et de transformation maligne (Barthélémy 2009). Dans le cas rapporté, la forme particulièrement agressive de la tumeur chez cette jeune patiente (récidive en 4 mois avec perforation des corticales et envahissement des parties molles) nous a orienté vers le diagnostic de TCG et un traitement radical de sa récidive. Conclusion : La TCG nécessite un diagnostic précoce et une exérèse en marges saines dès la première intervention afin de diminuer le risque de récidive et d'éviter des traitements plus mutilants. 